\title{
Prediction of imminent, severe deterioration of children with parallel circulations using real-time processing of physiologic data
}

\author{
Craig G. Rusin, PhD, ${ }^{a}$ Sebastian I. Acosta, PhD, ${ }^{a}$ Lara S. Shekerdemian, MD, ${ }^{b}$ Eric L. Vu, MD, \\ Aarti C. Bavare, MD, MPH, ${ }^{\mathrm{b}}$ Risa B. Myers, MS, ${ }^{\mathrm{d}}$ Lance W. Patterson, BS, ${ }^{\mathrm{a}}$ Ken M. Brady, MD, ${ }^{\mathrm{c}}$ and \\ Daniel J. Penny, MD, PhD, MHA ${ }^{\mathrm{a}}$
}

\begin{abstract}
Objectives: Sudden death is common in patients with hypoplastic left heart syndrome and comparable lesions with parallel systemic and pulmonary circulation from a common ventricular chamber. It is hypothesized that unforeseen acute deterioration is preceded by subtle changes in physiologic dynamics before overt clinical extremis. Our objective was to develop a computer algorithm to automatically recognize precursors to deterioration in real-time, providing an early warning to care staff.

Methods: Continuous high-resolution physiologic recordings were obtained from 25 children with parallel systemic and pulmonary circulation who were admitted to the cardiovascular intensive care unit of Texas Children's Hospital between their early neonatal palliation and stage 2 surgical palliation. Instances of cardiorespiratory deterioration (defined as the need for cardiopulmonary resuscitation or endotracheal intubation) were found via a chart review. A classification algorithm was applied to both primary and derived parameters that were significantly associated with deterioration. The algorithm was optimized to discriminate predeterioration physiology from stable physiology.
\end{abstract}

Results: Twenty cardiorespiratory deterioration events were identified in 13 of the 25 infants. The resulting algorithm was both sensitive and specific for detecting impending events, 1 to 2 hours in advance of overt extremis (receiver operating characteristic area $=0.91,95 \%$ confidence interval $=0.88-0.94)$.

Conclusions: Automated, intelligent analysis of standard physiologic data in realtime can detect signs of clinical deterioration too subtle for the clinician to observe without the aid of a computer. This metric may serve as an early warning indicator of critical deterioration in patients with parallel systemic and pulmonary circulation. (J Thorac Cardiovasc Surg 2016;152:171-7)

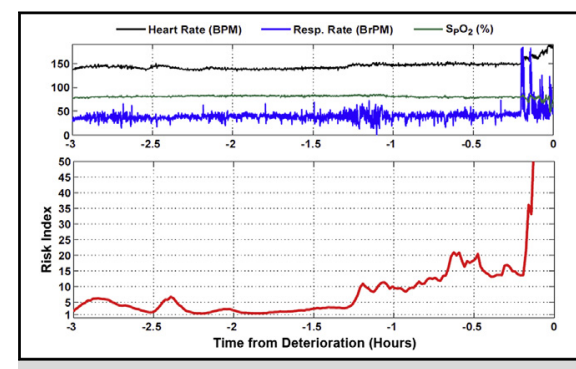

Vital signs and risk index as a function of time until deterioration.

\section{Central Message}

Critical deterioration events in children with parallel circulation can be predicted 1 to 2 hours before overt decompensation

\section{Perspective}

Acute clinical deterioration is common in patients with a hypoplastic ventricle and parallel circulation. Real-time mathematical transformations of standard physiological data can provide physicians with an early warning of such events allowing the opportunity for early interventions to be performed before the deterioration becomes life-threatening.

See Editorial page 3.
The management of newborns after surgical palliation for congenital heart disease is challenging. Hypoplastic left heart syndrome (HLHS), although accounting for $2 \%$ to

\footnotetext{
From the ${ }^{\mathrm{a}}$ Departments of Pediatrics-Cardiology, ${ }^{\mathrm{b}}$ Pediatrics-Critical Care, and ${ }^{c}$ Pediatrics-Anesthesia, Baylor College of Medicine, Texas Children's Hospital; and ${ }^{\mathrm{d}}$ Department of Computer Science, Rice University, Houston, Tex.

Research reported in this publication was conducted under a Scholar Award from the Pediatric Heart Network supported by the National Heart, Lung, and Blood Institute of the National Institutes of Health under Award U10HL068270 and the Rice University National Library of Medicine Training Program in Biomedical Informatics through the Keck Center of the Gulf Coast Consortia (T15LM007093).

Received for publication Oct 6, 2015; revisions received March 2, 2016; accepted for publication March 20, 2016; available ahead of print May 10, 2016.

Address for reprints: Craig G. Rusin, PhD, Department of Pediatrics-Cardiology, Baylor College of Medicine, Texas Children's Hospital, 1102 Bates St, Suite C 430.03, Houston, TX 77030 (E-mail: cgrusin@bcm.edu).

$0022-5223 / \$ 36.00$

Copyright (c) 2016 by The American Association for Thoracic Surgery http://dx.doi.org/10.1016/j.jtcvs.2016.03.083
}

$3 \%$ of all congenital heart disease, ${ }^{1}$ is responsible for up to $25 \%$ to $40 \%$ of all neonatal cardiac deaths. ${ }^{2-4}$ The Single Ventricle Reconstruction trial demonstrated that $87 \%$ of deaths of subjects undergoing palliation for HLHS occurred before the stage 2 palliative surgery, when the parallel circulation is replaced with a cavopulmonary shunt. ${ }^{5}$ As a result, these infants are monitored intensely and continuously. The reason for this is simple: the sooner the care team can detect that a patient is deteriorating, the quicker they can intervene to prevent

Scanning this QR code will take you to the article title page.

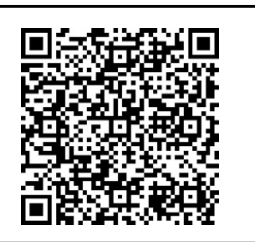




$$
\begin{aligned}
& \text { Abbreviations and Acronyms } \\
& \mathrm{BCM}=\text { Baylor College of Medicine } \\
& \mathrm{CPR}=\text { cardiopulmonary resuscitation } \\
& \text { CVICU }=\text { cardiovascular intensive care unit } \\
& \mathrm{ECG}=\text { electrocardiogram } \\
& \text { ECMO }=\text { extracorporeal membrane oxygenation } \\
& \text { HLHS = hypoplastic left heart syndrome } \\
& \text { ICU = intensive care unit } \\
& \text { IRB = institutional review board } \\
& \mathrm{ROC}=\text { receiver operating characteristic } \\
& \mathrm{SpO}_{2}=\text { peripheral capillary oxygen saturation } \\
& \mathrm{TCH}=\text { Texas Children's Hospital }
\end{aligned}
$$

catastrophic events from occurring. Recent evidence suggests that vigilant monitoring improves survival of patients with a single ventricle after stage 1 palliation.,

One of the most significant problems encountered while caring for these infants is that detecting impending deterioration can be difficult, even for experienced physicians, because current monitoring technologies are not optimized for this population. For example, in the cardiac intensive care unit (ICU), existing patient monitoring technologies may be confounded by baseline abnormalities of standard vital signs, such as pulse-oximetry, arterial blood pressure, and electrocardiogram. Thus, even when flow to the parallel pulmonary and systemic circulations is optimally balanced, patients are cyanotic. Pulmonary runoff from a systemic to pulmonary shunt can cause low diastolic arterial blood pressure. Cardiac conduction abnormalities can cause the ST segments to be elevated or depressed, so routine electrocardiogram (ECG) monitoring may not be useful to discriminate the status of myocardial perfusion. What is needed is a patient-monitoring system that is specificity optimized to detect problems in the unique physiology of patients with parallel systemic and pulmonary circulation, rather than for the general patient population.

The purpose of this study was to develop a new metric (a risk index) that is derived from continuous physiologic measurements, and predictive of imminent deterioration for subjects with parallel systemic and pulmonary circulations, before stage 2 palliation. Although the present study design is similar to that used in a traditional observational, multivariate regression analysis of factors related to deterioration, the process of predictive model development is fundamentally different. Rather than test for physiologic differences between subjects who experience a deterioration and those who do not, we assert that physiology immediately before deterioration is abnormal, and that physiology not in close proximity to deterioration events is stable. From this assertion, a classification model is constructed to maximize the recognition of predeterioration physiology from stable physiology based on recorded data. Our risk index is, therefore, a measure of how similar a patient's current physiology is to physiology that occurs just before critical deterioration. Real-time utilization of this index may provide the opportunity for clinicians to perform early interventions before deterioration becomes life-threatening, potentially affecting patient morbidity and mortality.

\section{METHODS \\ Patient Cohort and Data Collection}

A 1-year prospective observational study was conducted of infants who underwent surgical palliation at Texas Children's Hospital (TCH) in 2013. Approval was obtained from the institutional review board (IRB) at the Baylor College of Medicine (BCM) with a waiver of consent before the start of the study. Eligible subjects had any anatomic diagnosis of either a morphologic left or right ventricle, provided their early neonatal palliative surgery resulted in a mixing lesion with ventricular outflow to both pulmonary and systemic circulations. Subjects were enrolled on admission to the cardiovascular ICU (CVICU) immediately following early neonatal palliation, and enrollment continued until stage 2 palliation was performed (ie, the interstage period). All eligible subjects were enrolled and included in the analysis. Instances of critical deterioration were defined as the need for cardiopulmonary resuscitation (CPR) or endotracheal intubation, and were found via a chart review. Events were verified using physiologic recordings from patients.

Physiologic data were captured continuously from every monitored bed in the CVICU and the cardiology inpatient units using the Sickbay Platform (Medical Informatics Corp, Houston, Tex) for the duration of the study. Recorded data included high-resolution waveforms (eg, 4 ECG leads [240 Hz], chest impedance [120 Hz], vascular pressures [60 Hz]), and low-resolution vital signs measured intermittently or calculated by the bedside monitor at $0.5 \mathrm{~Hz}$ (eg, heart rate, respiration rate, arterial oxygen saturation $\left[\mathrm{SpO}_{2}\right]$, ST segments, arterial pressures). The data were stored on site for analysis, and automatically de-identified and coded by the Sickbay system to prevent the release of protected health information. Data analysis and model development were conducted using the Matlab (The MathWorks, Natick, Mass) programming environment. Data captured while a patient was on extracorporeal membrane oxygenation (ECMO) were excluded from the analysis.

\section{Selecting Input Variables to the Algorithm}

Candidate input variables to the algorithm were selected by a team of clinical experts based on physiologic rationale. This was done by delineating the etiologies of deterioration in these patients and then identifying measurable physiologic parameters that may be perturbed by these etiologies. Etiologies theorized to cause the observed events included cardiac ischemia, acute heart failure, shunt thrombosis, and changes in the systemic to pulmonary flow ratio due to changes in resistance of the respective vascular circuits. For each of these etiologies, a list of measurable candidate physiologic variables was generated. The initial candidate physiologic variables included heart rate, heart rate variability, arterial blood pressure (systolic, diastolic, and mean), common atrial pressure (systolic, diastolic, and mean), ST segment elevation, ST segment variability, respiratory rate, respiratory rate variability, $\mathrm{SpO}_{2}$, central venous pressure, core temperature, toe temperature, and core-toe temperature difference. This comprehensive list was then reduced by excluding measurements that were unlikely to be consistently present during a deterioration event, given the time frame of when most of the events were observed. For each remaining variable, a correlation coefficient was calculated between the values observed in the predeterioration and control data. The total number of input variables was limited to 6 so as to minimize model overfitting. The 6 variables with the largest magnitude correlation coefficients were included in the model. The final list of inputs included variables directly sampled from the monitor and those that are derived from the primary monitored 
data: heart rate, $\mathrm{SpO}_{2}$, respiration rate variability, beat-to-beat heart rate variability, ST segment from lead V1, and variability of the 3-dimensional ST segment vector.

Cardiac ischemia may be a prominent feature of the decompensation of infants with parallel circulation, but it is difficult to quantify with ECG monitoring because of conduction abnormalities that confound the predictive value of individual ST segment measurements. To overcome this limitation, we quantified the variability of the ST segment vector, which was constructed in 3 dimensions from the quasi-orthogonal leads $\mathrm{V}_{5}$, II, and aVL. The $x, y$, and $z$ coordinates of the instantaneous ST vector can be geometrically derived from the Einthoven triangle:

$$
\begin{aligned}
& \mathrm{ST}_{\mathrm{x}}=V_{5} \sin \left(30^{\circ}\right) \\
& \mathrm{ST}_{\mathrm{y}}=a V L \times \cos \left(30^{\circ}\right)+I I \times \cos \left(60^{\circ}\right)+V_{5} \times \sin \left(30^{\circ}\right) \\
& \mathrm{ST}_{\mathrm{z}}=a V L \times \sin \left(30^{\circ}\right)-I I \times \sin \left(60^{\circ}\right)
\end{aligned}
$$

where $\mathrm{V}_{5}$, II, and $\mathrm{aVL}$ are the values of the ST segments of the associated ECG leads. The movement of the ST vector was measured as the distance traveled by the tip of the vector over 30 -second intervals. This interval was chosen to remove the rapid, nonphysiologic changes in ST displacement generated by the GE monitors (General Electric, Fairfield, Conn) and because providers in the expert clinical panel reported the anecdotal occurrence of low-frequency changes in the ST segment, which are clinically viewed in 30 minute moving windows on the GE screen.

\section{Classification Algorithm Construction}

The physiologic data obtained from study subjects were separated into study (predeterioration) and control (nondeterioration) data sets. The study data set included all physiologic data recorded in the time interval starting 2 hours before each deterioration event and ending 1 hour before the event. The control data set included all physiologic data recorded more than 12 hours before or 24 hours after a deterioration event. If a subject did not experience a deterioration event, then all of the patient's physiologic measurements were included in the control data set.

Data recorded between 1 hour before deterioration and 24 hours after a deterioration event were not included in the classification model. Although this may be counterintuitive (physiology immediately preceding deterioration is most likely to be distinct from healthy physiology), there are 2 reasons to exclude these data. First, the acute decompensation state is not likely to be subtle, and is therefore likely to be recognized by the care providers, making event detection during this time window of limited clinical value. Second, the recognition of the predeterioration state by the care providers increases the likelihood of medical interventions that would distort the underlying physiology. Such interventions may potentially corrupt the model optimization process, creating an algorithm that recognizes the application of specific clinical interventions rather than physiologic precursors to critical deterioration. Similarly, deterioration events that occurred while the infant was mechanically ventilated also were not included in the model development process, as the ventilator could potentially introduce nonphysiologic characteristics into the study data set.

A multivariate logistic regression model of the form

$$
\ln \left(\frac{p}{1-p}\right)=\beta_{0}+\sum_{j=1}^{J} \beta_{j} \frac{x_{j}-\mu_{j}}{\sigma_{j}}
$$

was selected as the basis of the classification algorithm, where $p$ is the probability that the input metrics $\left(x_{1}, x_{2}, \ldots, x_{J}\right)$ belong to the study data set, $\mu_{j}$ is the mean value of $x_{j}$ over the data set, and $\sigma_{j}$ is the standard deviation of $x_{j}$. The input metrics to the model were computed from the raw physiologic observations, $y_{j}(t)$, averaged over a 10 -minute window. The baseline values were removed from the raw physiologic metrics before model optimization

$$
x_{j}(t)=y_{j}(t)-y_{j}^{\text {base }}(t)
$$

where $y_{j}^{\text {base }}(t)$ is the baseline value of metric $j$ computed as the average of $y_{j}(\mathrm{t})$ over a window of time from 6 hours before time $t$. The regression coefficients, $\beta_{j}$, were found using least squares optimization, so as to maximize the ability of the algorithm to separate the study data set from the control data set. This produced an algorithm that was tuned to recognize only those physiologic patterns that occur 1 to 2 hours before the onset of cardiorespiratory deterioration.

A risk index was derived from the logistic regression model by normalizing it to the average risk of deterioration for this population. A value of 1 represents that the infant is at average risk of having a critical deterioration event in the next few hours, whereas a value of 2 represents that the infant is at twice the average risk of deteriorating. The index can be continuously determined from the physiologic data of the patients and can be used to provide a calibrated measure of the risk of deterioration in the near future.

\section{Characterization of Algorithm Performance}

The area under the receiver operating characteristic (ROC) curve was used as the measure of the performance of the predictive model. ${ }^{8}$ Tenfold cross-validation was used to validate the predictive model, whereas numerical bootstrap aggregation was used to determine the statistical precision of the performance of the predictive algorithm. ${ }^{9-11}$ Bootstrapping was implemented using the perfcurve function provided by Matlab, with 1000 bootstrap replicas. This function returns the confidence bounds for the area under the ROC curve.

\section{RESULTS \\ Patient Characteristics}

Twenty-five infants were included in this cohort. Their anatomical characteristics and surgical procedures are presented in Table 1 . Of the 25 subjects, 13 had 1 or more cardiorespiratory deterioration events at some point during their interstage period (average $140 \pm 53$ days), with a total of 20 observed events, occurring at a mean of $48 \pm 40$ days after the initial surgery. Sixteen $(73 \%)$ events were considered to be primarily respiratory in nature, whereas $6(27 \%)$ were considered cardiac events. There were 4 hospital deaths in the cohort. Most of the patients who were identified for the study remained hospitalized until their second stage of surgical palliation (bidirectional Glenn). As a result, we were able to capture complete high-resolution physiologic recordings for almost all of the patients in this cohort, resulting in more than 72,000 hours of physiologic data.

\section{Predictive Performance of Algorithm}

The coefficients of the algorithm were optimized to maximize the recognition of physiologic features most associated with the time period just before the critical deterioration event (Table 2). Elevated heart rate was found to have the largest coefficient, indicating that it has the largest contribution to recognizing precursors of cardiorespiratory deterioration. In order of descending significance: decreased respiration rate variability, decreased $\mathrm{SpO}_{2}$, increased ST segment variability, ST segment depression, and decreased heart rate variability. The performance curves for the optimized model are shown in Figure 1. The ROC area for the model was calculated to be 0.91 
TABLE 1. Breakdown of the study cohort by anatomical characteristics and type of early neonatal palliation

\begin{tabular}{|c|c|c|c|c|}
\hline \multicolumn{5}{|c|}{ Anatomic characteristics } \\
\hline & Patients & Events & Duration, $d$ & Survival \\
\hline Morphologic left ventricular hypoplasia & 16 & 16 & $110( \pm 52)$ & $12(75 \%)$ \\
\hline Hypoplastic left heart syndrome & 13 & 13 & $108( \pm 36)$ & $10(77 \%)$ \\
\hline Heterotaxy with pulmonary atresia & 1 & 0 & 148 & $1(100 \%)$ \\
\hline Unbalanced atrioventricular canal & 1 & 1 & 97 & $1(100 \%)$ \\
\hline Double outlet right ventricle & 1 & 2 & - & $0(0 \%)$ \\
\hline Morphologic right ventricular hypoplasia & 9 & 4 & $176( \pm 34)$ & $9(100 \%)$ \\
\hline Tricuspid atresia & 5 & 3 & $168( \pm 59)$ & $5(100 \%)$ \\
\hline Pulmonary atresia & 3 & 1 & $189( \pm 57)$ & $3(100 \%)$ \\
\hline Double inlet left ventricle & 1 & 0 & 179 & $1(100 \%)$ \\
\hline \multicolumn{5}{|l|}{ Stage 1 (neonatal) palliation } \\
\hline Norwood with Blalock-Taussig shunt & 9 & 11 & $92( \pm 4.5)$ & $6(67 \%)$ \\
\hline Norwood with Sano shunt & 5 & 2 & $111( \pm 43)$ & $5(100 \%)$ \\
\hline Hybrid Norwood & 1 & 0 & 167 & $1(100 \%)$ \\
\hline Pulmonary artery band & 5 & 3 & $167( \pm 15)$ & $4(80 \%)$ \\
\hline Blalock-Taussig shunt & 5 & 1 & $194( \pm 56)$ & $5(100 \%)$ \\
\hline Overall & 25 & 20 & $140( \pm 53)$ & $21(84 \%)$ \\
\hline
\end{tabular}

The event column indicates the number of deterioration events observed by patient class. Some subjects experienced more than 1 deterioration event. Subjects were enrolled over the course of 1 year.

with a $95 \%$ confidence interval of 0.88 to 0.94 , indicating good performance in separating characteristics associated with the predeterioration data from control measurements. The sensitivity, specificity, and the positive likelihood ratio were calculated as a function of the choice of alerting threshold (Figure 1, $C$ and $D$ ) to determine the optimum operating threshold for using the index. For a threshold of 3 , the positive likelihood ratio is 10 , whereas the sensitivity is 0.81 and the specificity is 0.84 . For a threshold of 10 , the positive likelihood ratio is 16 , and the sensitivity is 0.40 and the specificity is 0.97 .

The risk index was calculated over time to verify its behavior in a subject who experiences a critical deterioration (Figure 2). In the hours preceding the event, the index vacillates between normal values (less than 1 ) and 3 , which indicates a threefold likelihood of critical deterioration within the subsequent 2 hours. In the 90 minutes preceding the event, the index increases progressively to a value of 18.7, seen 30 minutes before the observed event. Thus, the continuous reporting of the risk index showed a pattern of progressive decompensation from which 2 things can be clearly concluded: first, the physiology analyzed in this

TABLE 2. Parameters, associated coefficients, and statistical significance of the optimized logistic regression model

\begin{tabular}{lcr}
\hline \multicolumn{1}{c}{ Input parameter } & Coefficient & $\boldsymbol{P}$ value \\
\hline Heart rate & 1.06 & $<.001$ \\
Respiratory rate variability & -0.46 & $<.001$ \\
ST segment (V1) & -0.12 & .045 \\
$\mathrm{SpO}_{2}$ & -0.32 & $<.001$ \\
$\mathrm{~N}^{\mathrm{N}}$ heart rate variability & -0.10 & .052 \\
ST segment variability & 0.16 & .017 \\
\hline
\end{tabular}

$\mathrm{SpO}_{2}$, Peripheral capillary oxygen saturation. patient showed a pattern that was distinct from a cohort with similar physiology, and second, that this pattern is significantly associated with predeterioration physiology in the same cohort.

To estimate how far in advance the algorithm could be used to detect an impending event, its performance was evaluated as a function of time from deterioration (Figure 3). Each data point in the figure represents the ROC area of the predictive algorithm calculated using a 1-hour block of time, taken at different time points relative to the event. Thus, the point $\mathrm{t}=0$ hours represents the performance of the algorithm with data obtained between 0 and 1 hour before deterioration. By design, the algorithm has the highest performance approximately 1 to 2 hours before deterioration (ROC Area $=0.91$ ). As the forecasting time was increased, the performance of the algorithm was observed to decrease. Approximately 4 to 5 hours before a deterioration event, the ROC area was found to be close to 0.5 , indicating that the algorithm could no longer discriminate between predeterioration data and nondeterioration measurements.

The distribution of all measurements of the risk index is not Gaussian, as it is constructed as an odds ratio that is bounded at 0 with a median at 1 (Figure 4). The most common observed value was 0.6 . Risk index values greater than or equal to 3.3 are observed only $10 \%$ of the time, whereas values of risk index greater than or equal to 10 are observed only $2 \%$ of the time in this population.

\section{DISCUSSION}

The main result of this study is demonstration of a classification model that can discriminate a state of predeterioration from a state of stability on subjects with parallel 

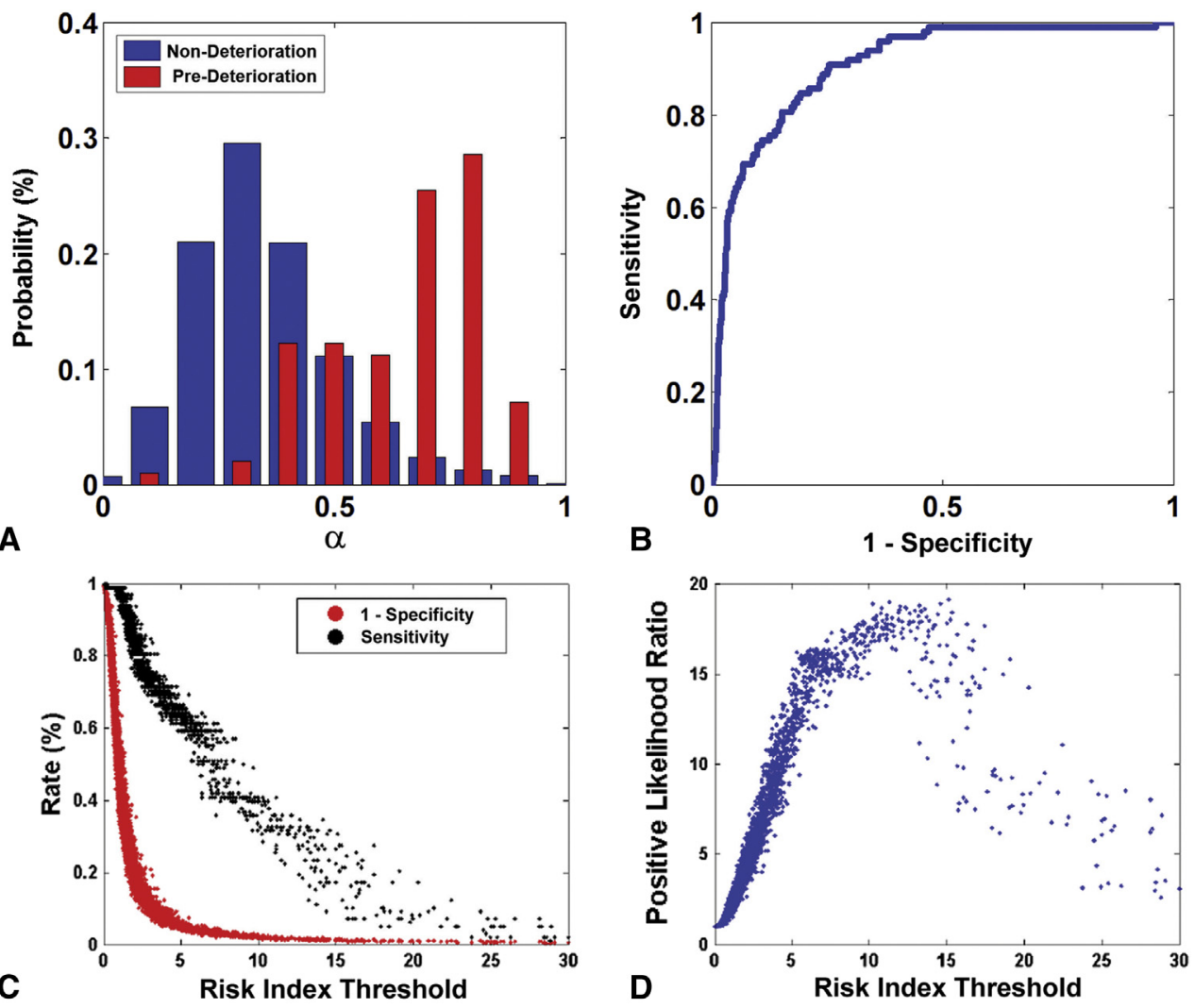

FIGURE 1. Performance of the optimized logistic regression model. A, Histogram of the optimized classification algorithm for the control and predeterioration data sets projected on the axis of optimal separation $(\alpha)$. B, The receiver operating characteristic curve for the optimized classification algorithm (ROC Area $=0.91)$. C, The sensitivity and specificity for the optimized classification model as a function of the choice of risk index threshold. D, The positive likelihood ratio as a function of the choice of risk index threshold.

circulatory anatomy and mixing physiology. Standard monitoring for these patients has a limited ability to discriminate between these states due to abnormalities of baseline vital signs when compared with patients who have normal serial circulation. The intent of real-time classification of patient physiology is to provide a rapid detection of impending clinical deterioration giving providers
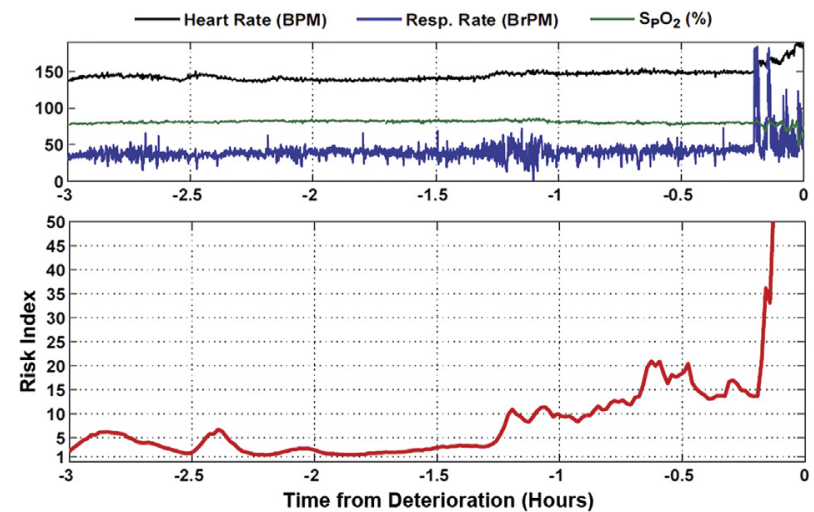

FIGURE 2. Vital signs (top) and the risk index (bottom) as a function of time until a critical deterioration event $(\mathrm{t}=0) . B P M$, Beats per minute; $\operatorname{BrPM}$, breaths per minute. increased time to assess and intervene. The Risk Index presented here demonstrated sensitivity and specificity for identification of the predeterioration state in subjects with parallel circulation in the 1- to 2-hour window before the deterioration event. Detailed analysis of the performance of the algorithm indicates that distinctive pathological features seem to appear approximately 5 hours before

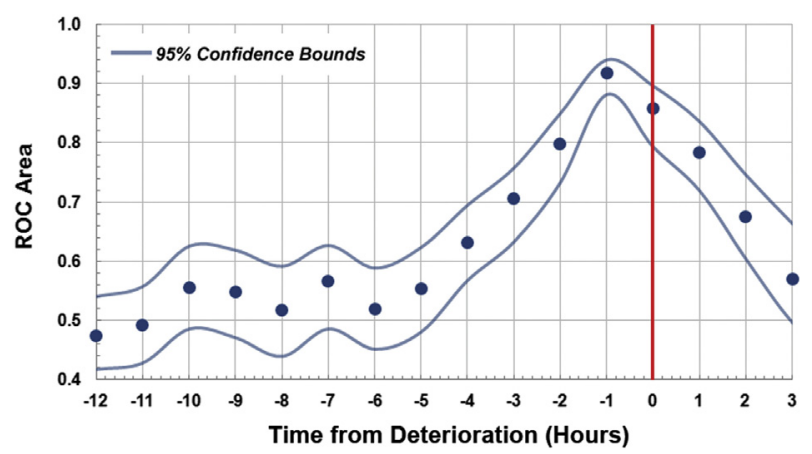

FIGURE 3. Performance of the predictive model as a function of time from the start of a cardiorespiratory deterioration event. Maximum forecasting time of the model is between 4 and 5 hours. ROC, Receiver operating characteristic. 

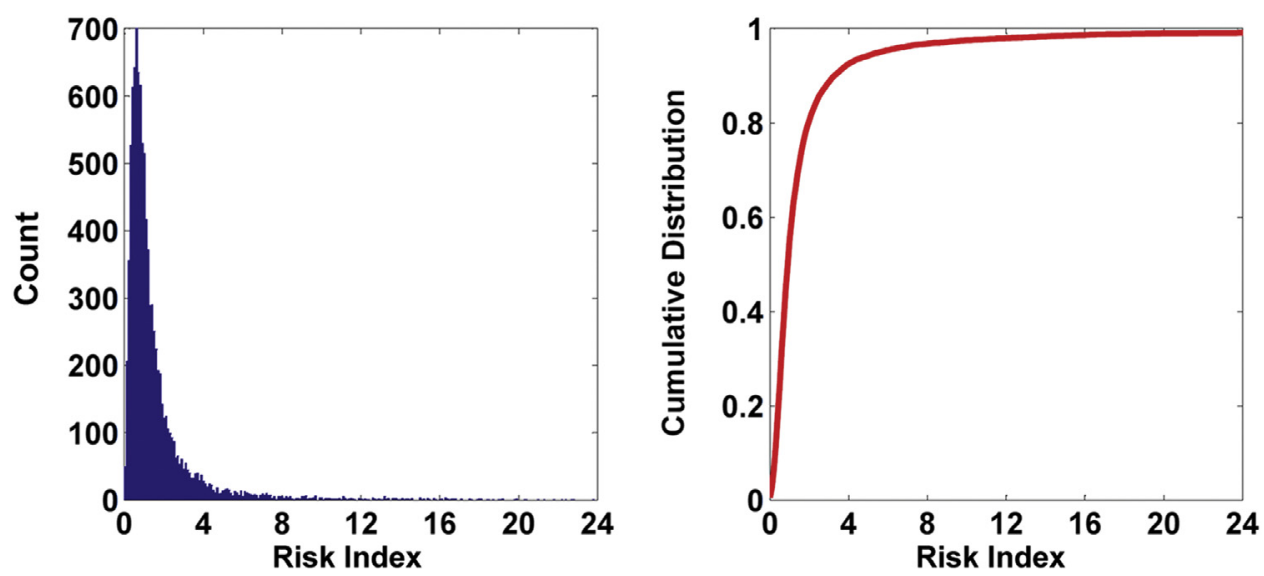

FIGURE 4. (Left) The observed distribution of the risk index metric for all subjects. The most common value of risk index was found to be 0.6. (Right) The cumulative distribution function of the risk index metric for all subjects. The prevalence of the metric was observed to be $50 \%$ for values $>1,10 \%$ for values $<3.3$, and $1 \%$ for values $>20$.

deterioration; however, such features are difficult to accurately and consistently recognize at this time scale with this data set.

If this risk exposure were evenly distributed in the cohort, then every subject would spend approximately 30 minutes a day with a risk index greater than 10 (Figure 4). However, it is reasonable to hypothesize that subjects with greater severity of illness have more exposure to predeterioration physiology, whether or not an actual event occurs. Further, it is a logical hypothesis that subjects who deteriorated also experienced a higher burden of predeterioration physiology exposure.

Several studies have been published describing different predictive risk scores for different clinical events and populations. ${ }^{12,13}$ Churpek et $\mathrm{al}^{14}$ shows that cardiac arrest events could be anticipated in adult population in the setting of a cardiac ward using vital sign data. Parshuram et $\mathrm{al}^{15}$ and Duncan et $\mathrm{al}^{16}$ developed and measured the performance of the bedside pediatric early warning score for determining the need for up-transfer to an ICU for hospitalized pediatric patients. McLellan et $\mathrm{al}^{17}$ described the performance of an algorithm to estimate the likelihood of arrest in children with heart disease using data from a physical examination for daily care management. There are also several published categorical risk metrics for children that can be evaluated at the time of ICU admission: the Pediatric Risk of Mortality (PRISM) and Pediatric Index of Mortality (PIM) scoring systems. ${ }^{18,19}$ PRISM and PIM focus on providing risk stratification models for benchmarking outcomes. These studies have several common features. Each study used a logistic regression model with a combination of vital signs and physical examination results to estimate risk. Data were manually collected by staff at intervals that were typically several hours long. Each study focused on estimating the risk of deterioration in the next 24 to 48 hours to assess the need for changes in daily management of care. However, none of these studies have addressed the need to improve the real-time detection of precursors to deterioration to improve the clinical response when events do occur. To our knowledge, no study to date has focused on detecting precursors to deterioration in children with congenital heart disease and parallel circulations for both ICU and non-ICU admissions.

The risk index has several advantages over traditional risk scores. Because the risk index is derived from continuous physiologic data, it can be updated automatically, continuously, and in real-time without affecting existing clinical workflow. Because the index was derived using all of the physiologic data generated by the infant during hospitalization, the metric is applicable in both ICU and non-ICU environments. Furthermore, the inputs to the model are calibrated to an individual subject, relative to a baseline value for each individual (Equation 3). This is important because the baseline physiologic parameters can vary widely between subjects, and for this complex population there is little consensus as to what constitutes "normal" or even "acceptable" for a given physiologic parameter.

The risk index has the potential to provide a highly sophisticated decision support tool to clinicians. The physiologic inputs into the index were selected to be sensitive to different mechanisms of patient decomposition. Therefore, although an increase in the risk index will not indicate a priori what type of clinical intervention may be required, the index will signal the need for additional medical surveillance and possibly additional diagnostic investigations, such as an echocardiogram or arterial blood sampling. The preclinical detection of impending deterioration allows time for a thorough assessment to be performed, so that any necessary interventions could be applied proactively. For example, if it is shown to be robust in larger groups of patients, an increase in the index may be an indication for priming an ECMO circuit or mobilizing the ECMO team, so that valuable time can be 
saved, in the event of serious deterioration. Furthermore, restoration of the index to baseline levels is likely to be an indication of successful therapeutic and resuscitative measures.

There were several limitations of this study. The study cohort is small $(\mathrm{n}=25)$ and all cohort members were from a single center. Additionally, there were only a small number of deterioration events that were observed $(n=20)$. Although data collection for this study was done prospectively, the data analysis and model development was done retrospectively. This approach was taken to ensure that the data recorded from the cohort reflected current standard of care practices for this population. Although unlikely, it is also possible that clinicians could have potentially anticipated these deterioration events and started interventions during the 1- to 2-hour time period before the event. Because of these limitations, future studies are required on independent cohorts to establish the true clinical performance of the algorithm at forecasting imminent deterioration events in this population. Studies on the performance of the algorithm to anticipate "near codes" are also required to demonstrate to what extent the risk index changes before such events.

Although these limitations are real, the performance of the optimized model is still significant. Through the use of bootstrap aggregation, we were able to efficiently use the limited data we have to both develop and validate the model. All physiologic input parameters provide a statistically significant contribution to the risk index. The classification performance of the optimized model (ROC Area $=0.91)$ is above the generally accepted threshold of 0.9 considered the minimum for clinical viability.

\section{CONCLUSIONS}

This study demonstrates that there exist recognizable physiologic precursors of acute cardiorespiratory deterioration in patients with parallel circulations. Although it can be difficult to recognize these precursors with current bedside monitoring technologies, real-time mathematical transformations of standard physiologic data can greatly enhance the ability to identify the onset of deterioration.

\section{Conflict of Interest Statement}

Craig Rusin is the cofounder of Medical Informatics Corp. Medical Informatics provided no funding and/or support for the study. All other authors have nothing to disclose with regard to commercial support.

\section{References}

1. Barron DJ, Kilby MD, Davies B, Wright JG, Jones TJ, Brawn WJ. Hypoplastic left heart syndrome. Lancet. 2009;374:551-64.

2. Norwood WI, Kirklin JK, Sanders SP. Hypoplastic left heart syndrome: experience with palliative surgery. Am J Cardiol. 1980;45:87-91.

3. Welke KF, Shen I, Ungerleider RM. Current assessment of mortality rates in congenital cardiac surgery. Ann Thorac Surg. 2006;82:164-71.

4. Schwartz RS, Murphy JG, Edwards WD, Camrud a R, Vliestra RE, Holmes DR Restenosis after balloon angioplasty. A practical proliferative model in porcine coronary arteries. Circulation. 1990;82:2190-200.

5. Ohye RG, Schonbeck JV, Eghtesady P, Laussen PC, Pizarro C, Shrader P, et al. Cause, timing, and location of death in the Single Ventricle Reconstruction trial J Thorac Cardiovasc Surg. 2012;144:907-14.

6. Ghanayem NS, Allen KR, Tabbutt S, Atz AM, Clabby ML, Cooper DS, et al. Interstage mortality after the Norwood procedure: results of the multicenter Single Ventricle Reconstruction trial. J Thorac Cardiovasc Surg. 2012;144:896-906.

7. Hansen JH, Furck AK, Petko C, Buchholz-berdau R, Voges I, Scheewe J, et al. Use of surveillance criteria reduces interstage mortality after the Norwood operation for hypoplastic left heart syndrome. Eur J Cardiothorac Surg. 2012;41:1013-8.

8. Zou KH, O’Malley J, Mauri L. Receiver-operating characteristic analysis for evaluating diagnostic tests and predictive models. Circulation. 2007;115:654-7.

9. Efron B. Bootstrap methods: another look at the jackknife. Ann Stat. 1979;7:1-26.

10. Platt RW, Hanley J, Yang H. Bootstrap confidence intervals for the sensitivity of a quantitative diagnostic test. Stat Med. 2000;19:313-22.

11. Zhou XH, Qin G. Improved confidence intervals for the sensitivity at a fixed leve of specificity of a continuous-scale diagnostic test. Stat Med. 2005;24:465-77.

12. Robson M-AJ, Cooper CL, Medicus L, Quintero MJ, Zuniga S. Comparison of three acute care pediatric early warning scoring tools. J Pediatr Nurs. 2013;28: e33-41.

13. Smith ME, Chiovaro JC, O'Neil M, Kansagara D, Quiñones AR, Freeman M, et al. Early warning system scores for clinical deterioration in hospitalized patients: a systematic review. Ann Am Thorac Soc. 2014;11:1454-65.

14. Churpek MM, Yuen TC, Park SY, Meltzer DO, Hall JB, Edelson DP. Derivation of a cardiac arrest prediction model using ward vital signs. Crit Care Med. 2012 40:2102-8.

15. Parshuram CS, Duncan HP, Joffe AR, Farrell C, Lacroix JR, Middaugh KL, et al Multicentre validation of the bedside paediatric early warning system score: a severity of illness score to detect evolving critical illness in hospitalised children. Crit Care. 2011;15:R184.

16. Duncan H, Hutchison J, Parshuram CS. The pediatric early warning system score: a severity of illness score to predict urgent medical need in hospitalized children. J Crit Care. 2006;21:271-8.

17. Mclellan MC, Gauvreau K, Connor J. Validation of the cardiac children's hospi tal early warning score: an early warning scoring tool to prevent cardiopulmonary arrests in children with heart disease. Congenit Heart Dis. 2014;9:194-202.

18. Shann F, Pearson G, Slater A, Wilkinson K. Paediatric index of mortality (PIM): a mortality prediction model for children in intensive care. Intensive Care Med. 1997;23:201-7.

19. Pollack MM, Patel KM, Ruttimann UE. The Pediatric Risk of Mortality III-Acute Physiology Score (PRISM III- APS): a method of assessing physiologic instability for pediatric intensive care unit patients. J Pediatr. 1997;131:575-81.

Key Words: critical deterioration, single ventricle, predictive analytics 